\title{
The presence of a foreign body in the digestive tract - case reports and literature review.
}

\author{
Daniel Houser ${ }^{1}$, Mark H. Johnston ${ }^{2 *}$
}

Received: 04 March 2018; Accepted: 30 June 2018; Published online: 20 July 2018 DOI: https://doi.org/10.32391/ajtes.v2i2.3

\begin{abstract}
Background: The presence of foreign bodies in the digestive tract usually occurs in pediatric, adolescent and psychiatric patients. Current Protocols focus mainly on pediatricians and adults.

We present some cases mainly of feminine age. Most swallowed foreign bodies will harmlessly pass through the GI tract, but some will lead to health problems if they become lodged (too big to pass, such as a spoon, or small objects like a pill that adheres to a moist surface); traumatize the mucosa (sharp or pointed object, like a razor blade or pin), or cause burn-like illness (biologically active, such as a button battery or a medication patch) may cause problems.

which during X-Ray abdominal exfoliation show varying objects in the digestive tract at different levels. The following examinations highlighted the most accurate localization.

Asymptomatic clinical data continued to deteriorate, despite imaging evidence. This article discusses the challenges involved in the management of foreign troops in the digestive tract and the re-evaluation of literature Keywords: digestive tract, foreign body, management, pediatric patient
\end{abstract}

$\equiv{ }^{*}$ Corresponding author: Daniel Houser

E-mail: cryodoc@gmail.com

${ }^{1}$ Mark Johnston MD, Penn Medicine Lancaster General Health, Lancaster, PA, USA

${ }^{2}$ Daniel Houser MD, Uniformed Service University of Health Sciences, Bethesda, USA 


\section{Introduction}

Foreign body (FB) ingestion and food bolus impaction is a common reason for people of all ages to visit the emergency room. Although nearly $80 \%$ of FBs pass spontaneously, 20\% require endoscopic intervention and nearly $1 \%$ require a more invasive surgical procedure.

The majority of FB ingestion cases are in children between 6 months and 3 years. These cases are largely accidental, unwitnessed, and can be asymptomatic, suggesting that the incidence is actually higher than what is reported (Shah \& Mamula, 2018). Adults present more often with a food bolus impaction secondary to a pre-existing condition, such as eosinophilic esophagitis, strictures, malignancy, post-GI surgery, hiatal hernia, or achalasia (Ambe et al, 2012) (Yuan, 2017). Another subset of cases that require special attention is those of intentional ingestion. Incarcerated persons, psychiatric patients, and those with behavioral problems have a higher incidence of ingestion with multiple objects and recurring ingestions, and more often require intervention. In one study, $69 \%$ of cases from intentional FB ingestion required endoscopic intervention, and $22 \%$ required surgery (Dalal et al, 2013).

The most common objects ingested by children are coins, magnets, batteries, small toys, plastics, jewelry and buttons (Shar \& Mamula, 2018). Food impactions are more common in older children $(>10$ years) and true foreign bodies are more common in younger children (<10) (Sugawa et al, 2014). Adults more often present with food related ingestions, such as fish and chicken bones, chunks of meat, fruit seeds, and toothpicks. Other items may include metallic objects (ie rings) or dentures (Yuan et al, 2017) (Ambe et al, 2012) (ASGE, 2011). A study of intentional ingestions within the incarcerated population found a variety of objects such as pens, razorblades, utensils, hardware, bed springs, toothbrushes, glass, batteries, and various metal objects (Dalal et al, 2013). The location of impaction, perforation, or obstruction varies with object type, but not necessarily with age, and may be associated with physiological narrowing, underlying pathology, previous surgery, or congenital malformations (Geng et all, 2017) (Yuan et al, 2017) (Shah \& Mamula, 2018).

Common sites of acute angulation or narrowing are the following:

\section{Cricopharyngeus muscle (cervical esophagus)}

2. Aortic arch (thoracic esophagus)

3. Left main stem bronchus (thoracic esophagus)

4. GE junction

5. Pylorus

6. Duodenal sweep

7. Ileocecal valve

8. Anus

According to two large studies done in China, 82$87 \%$ of FBs requiring intervention become lodged between the pharynx and the distal esophagus, primarily in the cervical and thoracic esophagus (Geng et all, 2017) (Yuan et al, 2017). In a study focused on cases of intentional ingestion, nearly $50 \%$ were in the stomach (Dalal et al, 2013). Fortunately, only $20 \%$ require endoscopic intervention, less than $1 \%$ require surgery, and the rest pass spontaneously without complication (Yuan, 2017; Shah, 2018). Children make up the majority of FB ingestion cases in the U.S*. with approximately $85 \%$, however in two studies conducted in China, adults were found to have 
higher incidences of reported ingestions/impactions (Yuan, 2017; Geng, 2017).

*includes U.S. territories of Guam, Puerto Rico, American Samoa, U.S. Virgin Islands, District of Columbia, and the Federated States of Micronesia.

\section{Diagnosis}

\section{History and Physical Exam}

Initial workup of a suspected FB ingestion must begin with a thorough history and physical exam. Adults most often present with food impaction secondary to a pathologic process such as in eosinophilic esophagitis. Patients with FB symptoms may or may not be aware that they ingested something. In communicative children and adults, the exact details of when and what type of $F B$ was ingested may be conveyed to the physician. This information should be gathered from the caretaker in young children, mentally impaired, and older, non-communicative adults (Shah and Mamula, 2018). Additional information helpful to the determination of care include the size and configuration of the object to include a picture if possible, last oral intake, characterization of symptoms, and past medical history significant for gastrointestinal abnormalities, surgery, or disease. Inquiry of common FB associations should be made for patients who are not certain of an ingestion. $A$ history of prior dysphagia or odynophagia, presence of dental prosthetics, and a patient's occupation may help in diagnosing an unknown ingestion (tailors with needles).

In some cases, the patient may indicate the perceived location of the FB. This is not shown to have a strong association with the actual location and should not be relied on for diagnostic purposes (Connolly et al, 1992). Patients may present within hours of FB ingestion, however if unwitnessed or unbeknownst, may not present for days, months or years after ingestion (Geng, 2017). Often the precipitating factor is the onset of symptoms.

\section{Symptoms}

Symptoms may or may not be present, and this can prolong the time to treatment. This is especially pertinent for pediatric patients who may be completely asymptomatic. Children brought in by an adult who witnessed or suspected an ingestion should be assumed to have ingested a FB until it is completely ruled out. Fortunately, most patients present with symptoms, many of which may help to localize where the impaction is. Children most often present with vomiting, drooling, and dysphagia, while adults more often present with odynophagia, $F B$ sensation and sore throat. Other symptoms include retrosternal pain, abdominal discomfort, and respiratory symptoms of coughing, stridor, and choking (Jayachandra \& Eslick, 2013) (Geng et al, 2017) (Shah \& Mamula, 2018). In more severe pediatric cases, fever, aspiration pneumonia, and failure to thrive may indicate a prolonged ingestion period, perforation, or deep ulceration (Shah, 2018).

\section{Imaging}

Radiographs of the affected area are recommended in one or two planes to first, confirm diagnosis, and secondly to determine location, orientation, number, and size of the FB. Foreign bodies can be radiodense or radiolucent, and this should be considered before any imaging is done. Most large, dense FBs are easily identified on plain radiograph; however, small animal bones, wood, plastic, glass, and thin metal objects are difficult to distinguish. Although computed tomography (CT) is not the first choice for imaging FBs, it is recommended in circumstances of suspected complications or delayed presentation, where it should be the firstline mode of imaging (Shah, 2018). Where radiation 
exposure is a concern, point of care (POC) ultrasound may play a part. POC ultrasound is rarely cited as a commonly used method, however it has been recommended for use as an adjunct diagnostic tool to determine esophageal measurements, persistence of intraluminal air-fluid level, and direct $F B$ visualization (Singleton, 2017).

Metal detectors can be used to confirm a general location of a metal FB. Mainly used in children. Contrast studies, such as gastrograffin, are contraindicated due to the increased risk of aspiration and impaired visualization during endoscopy which is frequently the next step in care.

\section{Management}

\section{General}

The European Society of Gastrointestinal Endoscopy recommends early referral to the Emergency Department (ED) and evaluation with X-Ray for anybody suspected of a FB ingestion, even if asymptomatic (ESGE). Since $80-90 \%$ of ingested FBs pass without any difficulty, conservative treatment is recommended in the absence of significant symptoms, especially those that are blunt, short $(<6 \mathrm{~cm})$, and narrow $(<2.5 \mathrm{~cm})$ (Ambe, 2012). However, certain objects run a higher risk of complications and must be removed. Ingestion of batteries, magnets and sharp/pointed objects, or obstructive symptoms in adults and children are considered emergent and the FB must be removed as soon as possible (Shah, 2018; Ambe, 2012). Objects in the esophagus causing minimal symptoms or longer than $6 \mathrm{~cm}$ are considered urgent and should be removed within 24 hours. (Shah, 2018; Ambe, 2012). FBs more than $2.5 \mathrm{~cm}$ in diameter are non-urgent and may be removed if there is a clinical indication, or the patient elects to have it removed. (Shah, 2018; Ambe, 2012)

\section{Equipment}

The type of endoscopic equipment used is contingent upon the type of FB being removed. Overall, both flexible and rigid endoscopy are commonly used depending on where the impaction is located and additional considerations involved, such as the need to protect the airway, where rigid endoscopy may prevent the need for an over tube. Overall, there is a $98.3 \%$ success rate for removal of FBs by endoscopy (Geng, 2017). Over $85 \%$ of FBs were found by flexible endoscopy (Geng, 2017). Those not removed successfully were chicken, fish and pig bone FBs (31.6\%), metal FBs (21.1\%), and dental prostheses (15.8\%). (Geng, 2017). Foley catheter, fluoroscopic balloon extraction (Rosenfeld, 2018)

Rat-tooth and Alligator tooth forceps, polypectomy snares, polyp grasper, Dormier basket, biopsy forceps, retrieval nets, magnetic probes, friction fit adaptors, banding caps, transparent caps, endoscopy scissors have all been used successfully to remove FBs. Single and double balloon enteroscopes for children have also been used successfully (Shah, 2018).

\section{Food bolus impaction}

Food bolus impaction makes up the majority of esophageal obstruction cases in adults and should 


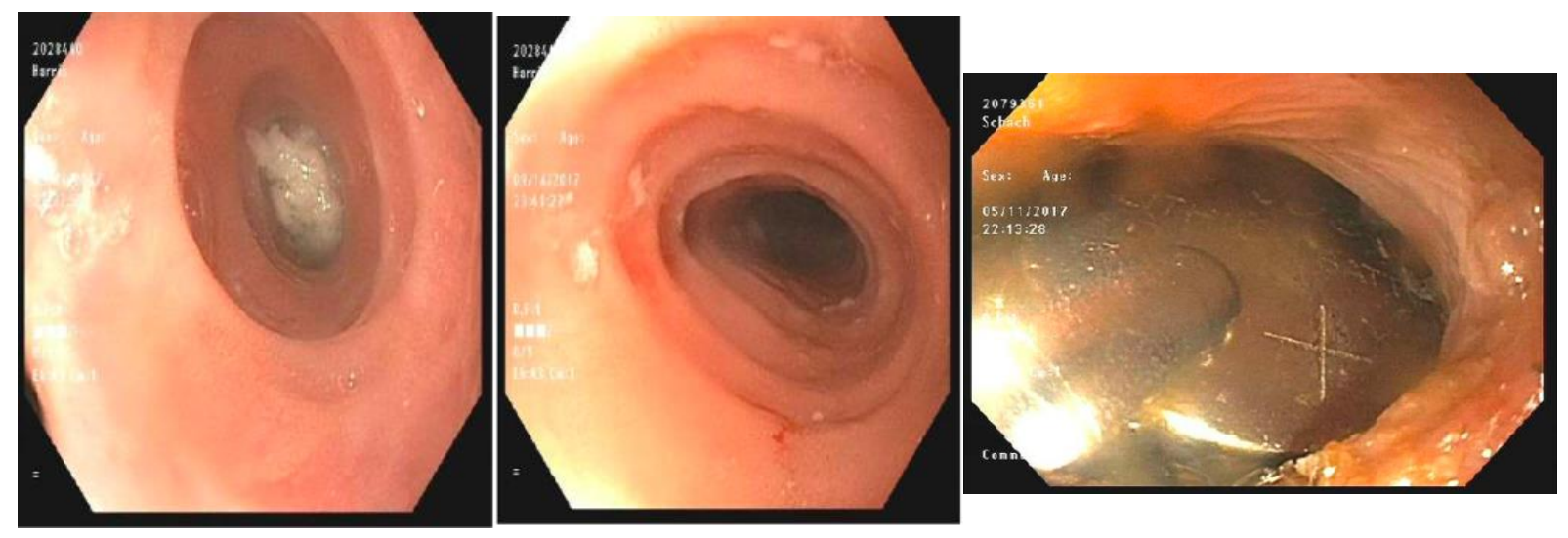

Figure 1 - Middle third of esophagus: Foreign body

Figure 2 - Eosinophilic esophagitis Figure 3 - Upper third of esophagus

be considered a medical emergency. Often, the patient is reasonably comfortable, in which case intervention can be postponed, as passage of the food bolus will sometimes occur spontaneously. Regardless, obstructions should be cleared within 24 hours to reduce the risk of complications. Although some studies encourage biplane radiographs immediately to determine the presence or absence of bones, the European Society of Gastrointestinal Endoscopy (2016) suggests that a more conservative approach be taken if the patient is asymptomatic, and only resort to radiographic imaging when bones are suspected. Underlying pathology is strongly associated with adult food bolus impaction and may be as high as

\section{Batteries}

Batteries pose a special risk when ingested and require immediate attention. The majority of cases are children under 5 and involve small, round disk or button batteries found in small electronics such as toys, remote controls, and watches (Rosenfeld, 2018). Although some batteries pass through the $\mathrm{GI}$ system without complication, immediate intervention, including attempted removal and at a minimum close monitoring, is recommended due to
93\% (Truskaite \& Dlugosz, 2016). A recent survey indicated that only $34 \%$ of gastroenterologists regularly perform a biopsy following removal of an esophageal food bolus, it is estimated that over 10,000 cases of eosinophilic esophagitis per year in the US may be missed as a result (Hiremath et al, 2017).

In another study, $40 \%$ of food bolus impactions were associated with an underlying diagnosis of eosinophilic esophagitis (Truskaite \& Dlugosz, 2016). ood in esophagus due to eosinophilic esophagitis Lithium battery in 5 years old child's esophagus (Figures 1-3).

the severity of possible complications. The most common of these are associated with esophageal impactions and include mucosal irritation, esophageal perforations, and in the most severe cases, hemorrhage due to trans-esophageal fistulas (TEF) impacting the aorta which often lead to death, especially in children under the age of 2 years (MMWR, 2012). Esophageal damage of any degree warrants hospitalization. 
Diagnosis of battery ingestion has been successfully made using plain radiographs in $94 \%$ of patients (Rosenfeld, 2018), and been able to identify batteries in the esophagus, stomach, small bowel, and colon. Patients with esophageal battery impaction may present with sore throat, coughing, respiratory difficulties, and drooling, which retrospective studies have found to lead to incorrect diagnosis of an upper respiratory tract infection, prolonging critical treatment and increasing the risk for severe complications, especially in young patients. Early identification and imaging to confirm the diagnosis is key to timely treatment of patients with potentially fatal impactions.

Patients with esophageal battery impactions should always receive intervention. Common interventions are fluoroscopic balloon extraction and rigid or flexible endoscopy, although Foley catheter extraction is also used. When extraction is not possible from the esophagus, pushing the battery into the stomach and attempting retrieval from there is an alternative method (ASGE, 2011). Batteries that enter the stomach, bowel, or colon are less likely to become lodged and should be managed non-operatively. Serial radiographs should be taken every 3-4 days to observe progression, and in the event of non-progression for more than 48 hours, surgical removal may become necessary (ASGE, 2011) (Rosenfeld, 2018).

\section{Blunt objects}

The most common blunt objects are coins in children in US and China studies, followed by bony FBs, plastic bars and iron nails (Geng, 2017).

(Figures 4-8) These should all be removed if in the esophagus, within $24 \mathrm{hrs}$ if asymptomatic, $2 \mathrm{hrs}$ if symptomatic or a button battery. If in stomach or below, follow if asymptomatic, remove if symptomatic (ESGE).

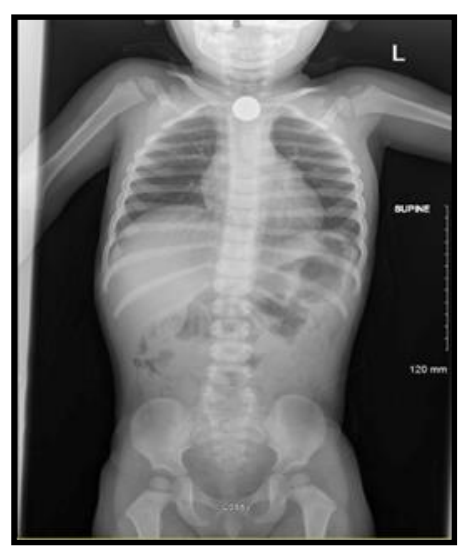

Figure 4 - Penny in 2 years old esophagus

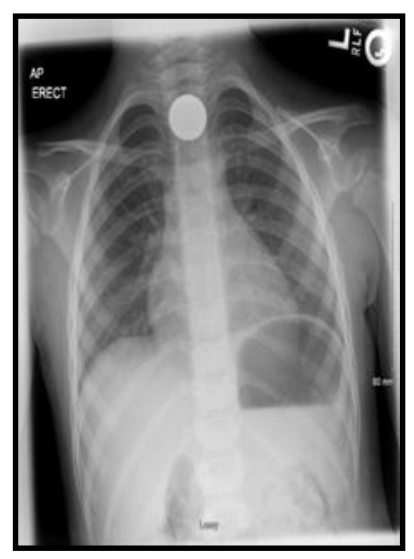

Figure 5 - Quarter stuck in 5 years old esophagus

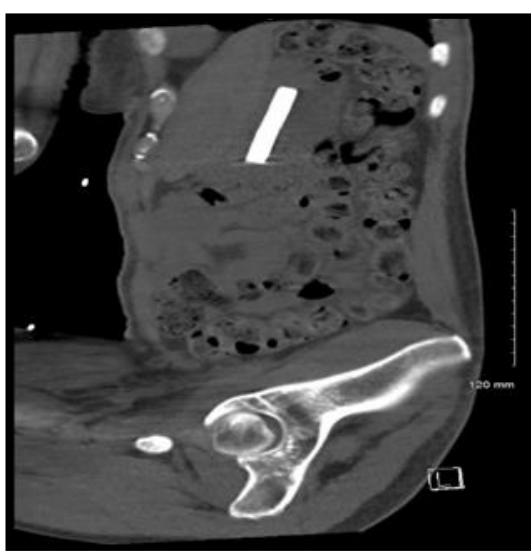

Figure 6 - DOMINO in stomach (CT sagittal view) 


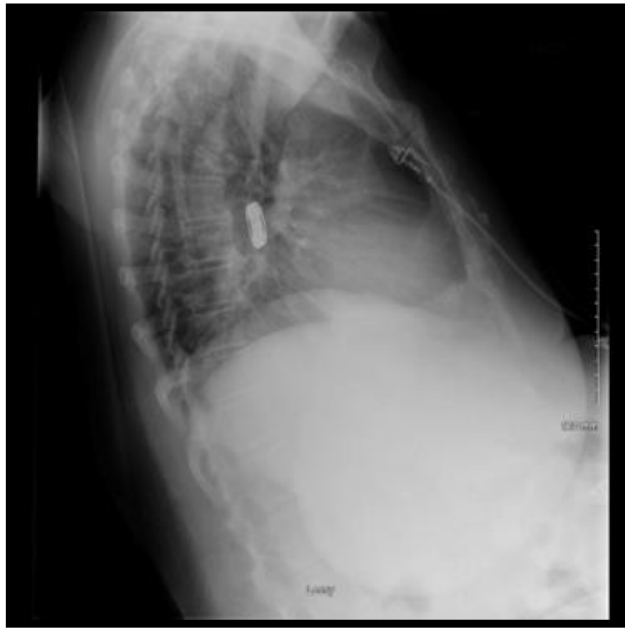

Figure 7 - Two buttons in the esophagus (lateral view)

\section{Sharp-pointed objects}

In Geng's series bony FBs made up $65 \%$ of cases and included fish, chicken, and duck bones (Geng, 2017)

\section{Long objects}

Objects longer than $6 \mathrm{~cm}$ should be removed endoscopically as they are unlikely to pass through the duodenal sweep (Pellerin, 1969). These include such objects as pens, pencils, toothbrushes, and eating utensils.

\section{Narcotic packets}

Concealment of illegal drugs via ingestion of balloons, condoms or wrapped in plastic has been reported in both children and adults. These are usually visible radiographically. Rupture or leakage of contents can be fatal. Thus, endoscopic removal is contraindicated. Surgical removal should be performed when the packets fail to pass or intestinal obstruction is apparent.

- One particular group of patients that pose a significant surgical challenge are $F B$ ingested in the anus for pleasure seeking

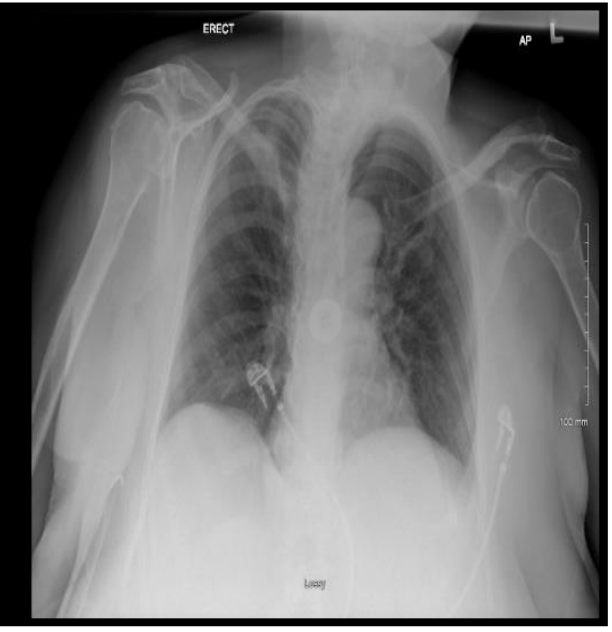

Figure 8 - Two buttons in the esophagus (AP view)

\section{reasons. These usually end up having} surgery...

- The other group of patients are those who repeatedly swallow just about any $F B$ to get surgical attention...

\section{Conclusion}

Foreign body ingestion and food bolus impaction is a relatively common presentation to the ED. Emergent removal should be performed for all esophageal food impactions, foreign bodies with evidence of complete esophageal obstruction, disk batteries in the esophagus and magnets within reach of an endoscope. Removal should also be performed for objects with a diameter of $2.5 \mathrm{~cm}$ from the stomach, sharp-pointed objects or those longer than $6 \mathrm{~cm}$. Removal of suspected drug containing packets should only be done surgically if packets are suspected to rupture or if they fail to progress radiographically. 


\section{References}

1. Geng, C., et al., Endoscopic management of foreign bodies in the upper gastrointestinal tract: a retrospective study of 1294 cases. Scand J Gastroenterol, 2017. 52(11): p. 1286-1291.

2. Shah, A. and P. Mamula, Foreign Body Ingestions: Approach to Management. Current Pediatrics Reports, 2018. 6(1): $p$. 57-66.

3. Ambe, P., et al., Swallowed foreign bodies in adults. Dtsch Arztebl Int, 2012. 109(50): p. 869-75.

4. Dalal, P.P., et al., Intentional foreign object ingestions: need for endoscopy and surgery. J Surg Res, 2013. 184(1): p. 145-9.

5. Morbidity and Mortality Weekly Report. (August, 2012). Injuries from batteries among children aged $<13$ years - United States, 1995-2010. Centers for Disease Control and Prevention. 61; 34.
6. Rosenfeld, E.H., et al., Battery ingestions in children: Variations in care and development of a clinical algorithm. J Pediatr Surg, 2018.

7. Hiremath, G., et al., Management of Esophageal Food Impaction Varies Among Gastroenterologists and Affects Identification of Eosinophilic Esophagitis. Dig Dis Sci, 2018.

8. Committee, A.S.O.P., et al., Management of ingested foreign bodies and food impactions. Gastrointest Endosc, 2011. 73(6): p. 1085-91.

9. Sugawa, C., et al., Endoscopic management of foreign bodies in the upper gastrointestinal tract: A review. World J Gastrointest Endosc, 2014. 6(10): p. 47581.

10. Pellerin D, Fortier-Beaulieu M, Gueguen J. The fate of swallowed foreign bodies: experience of 1250 instances of subdiaphragmatic foreign bodies in children. Progress in Pediatric Radiology 1969; 2: 286-302. 